\title{
Prehension movements and perceived object depth structure
}

\author{
UMBERTO CASTIELLO \\ University of Melbourne, Parkville, Victoria, Australia \\ CLAUDIA BONFIGLIOLI \\ Dipartimento di Psicologia, Bologna, Italy \\ and \\ KERRY BENNETT \\ La Trobe University, Burdoora, Victoria, Australia
}

\begin{abstract}
This study asked the question, "Will the motor pattern to a perceived two-dimensional (2-D) object differ from that same object when it is perceived as three dimensional (3-D)?" Subjects were required to reach and grasp an apple that could appear to be 2-D or 3-D. Two experimental sessions were conducted. In Condition A, the apple was initially perceived to be $2-\mathrm{D}$, but, for $20 \%$ of trials, it suddenly shifted to a 3-D apple at movement onset. In Condition B, the apple was initially perceived to be 3-D, but, for $20 \%$ of trials, it suddenly shifted to a 2-D silhouette of the same apple. For control trials, subjects grasped the perceived 2-D apple as if it were a disc (82\%), and they grasped the 3-D apple, as they would a normal apple, with a whole-hand grasp (86\%). For Condition A perturbed trials, there was a rapid change from a 2-D precision grip to a $3-\mathrm{D}$ whole-hand prehension, whereas the converse was true for the opposite perturbation. Peak acceleration was anticipated for Condition A perturbed trials but not for Condition B perturbed trials. These results indicate that the motor patterns we use in interacting with an object are strongly influenced by the way we perceive the object in real time, and that object affordances, such as dimension, can override the influence exerted by existing representations.
\end{abstract}

Information extracted from visual input is thought to be channeled differentially through identification/recognition and action pathways. At a functional level, this means that the "object descriptions that permit identification and recognition may be computed independently of the sets of descriptions that allow an observer to shape the hand appropriately to pick up an object" (Goodale \& Milner, 1992, p. 20). Evidence for such differential channeling has been largely provided by brain-damaged subjects. Particularly illustrative cases are those optic ataxic patients who are reported to be able to identify objects but are not able to shape the hand appropriately for objects of differing sizes (Jakobson, Archibald, Carey, \& Goodale, 1991). One explanation of this observation is that the activation of neural structures underlying mental representations for the object for the purposes of recognition does not equate with access to those structures that underlie the planning and activation of appropriate movement parameterization.

This work was supported by the Australian Research Council to U.C. and K.B. The authors thank Peter Wenderoth for his constructive comments on earlier versions of this manuscript, Enrico D'Amico (B $|\mathrm{T}| \mathrm{S} \mid$ s.r.l.) and M. Bulgheroni (BIOHELP) for technical support, Alessandro Allodi for his expert advice about the spotlighting procedure, and Sharon Harrup for her assistance with the figures. Correspondence should be addressed to U. Castiello, Department of Psychology, School of Behavioral Science, Faculty of Medicine, University of Melbourne, 3052 Parkville, VIC, Australia (e-mail:u.castiello@psych.unimelb. edu.au).
Using terminology slightly different from the "what" and "where" of Ungerleider and Mishkin (1982), Goodale and Milner refer to "what" and "how" pathways. Despite knowing "what" the object is, optic ataxic patients cannot operationalize "how."

The disruption observed to the "how" channels appears to reflect the inability to use neither on-line visual input nor representational knowledge to guide movement. This leads to the question of whether the dysfunction is purely to a visuomotor channel that ordinarily bypasses representational knowledge systems - a visually guided channel-or whether the dysfunction is to neural processes somewhere between this accessing and the action formulation stage-a vision and representational guided channel for action. In both cases, the function of recognition would not be compromised.

However, evidence supporting a dissociation between the visuoperceptual channels for identification and the visuomotor channels for action comes from a patient (D.F.) with visual form agnosia, resulting primarily from damage to areas 18 and 19, who could not identify objects or discriminate visual form (e.g., size, shape, orientation) but could reach to grasp objects of different sizes, scaling the grip appropriately to the object's dimensions (Goodale, Milner, Jakobson, \& Carey, 1991; Milner et al., 1991). Here, the information "afforded" (Gibson, 1969) by the object was efficiently and appropriately used by an action system. 
Distinct to the view that movement can be driven solely by perceptual influences is the idea that motor responses are influenced by cognitive knowledge. According to Klatzky, McCloskey, Doherty, Pellegrino, and Smith (1987), the motor system uses canonical descriptions based on structural attributes of the object to derive an association between motoric and object representations. In their study, a bidirectional relationship was established between categorical representations of hand configurations and object categories in memory. An object is viewed with the "hand's eye," and structural/functional attributes (e.g., size and "projection into depth") are mapped onto aspects (e.g., "prehensibility") that differentiate hand configurations. With such a theoretical stance, it can be supposed that there is less of a dissociation between channels subserving action and those subserving recognition, the on-line motor response resulting from interplay of both perceptual and more cognitive sources of information.

One means of testing the degree of interplay between visuomotor and mental representational systems in nonbrain-damaged subjects is to assess goal-directed movement to an object using paradigms that dissociate perception of a structural attribute (size, projection into depth, etc.) from its actual status/identity. A movement well suited for this purpose is the simple act of reaching to grasp an object; several studies have demonstrated that the kinematics of this action are sensitive to changes in object features such as size, position, and shape (Castiello, 1996; Jeannerod, 1981; see Bennett \& Castiello, 1994, for review). An obvious example is the change of maximum grip aperture that occurs with changes to object size. However, the reaching arm also shows changes to its velocity and deceleration patterns according to object size.

In studies that have dissociated perception of object size from actual size (i.e., size illusion studies), there is no clear consensus as to the degree to which perception of an object influences the motor action on that object. The movement parameter of "grip aperture," maximum distance between the index finger and thumb during a reaching to grasp action, does not appear to be "fooled" by size illusions, showing appropriate scaling for the actual, as opposed to the perceived, size of an object (Aglioti, DeSouza, \& Goodale, 1995; Brenner \& Smeets, 1996). In contrast, however, the parameter of "grip force" has been shown to change according to perceived size of an object, indicating some input from systems that have used the size information to infer object weight (Brenner \& Smeets, 1996).

The structural attribute of dimension has received little investigation as a potential modulator of movement organization. Yet it is clear that the degree to which an object projects into depth will determine the "prehensibility" (Klatzky et al., 1987) of the object, the extent to which the hand can close about it, and, thus, the type of grasp that would be utilized. In the present study, two main experiments were conducted to assess the effect of perceived, rather than actual, dimension (depth structure) on the reach-to-grasp movement. A "depth struc- ture illusion" was created by special lighting capable of making an object (in this case, an apple) appear two dimensional (2-D). For some trials, perceived depth structure was changed unexpectedly at movement onset, making it possible to assess whether or not the system continued to adopt the same movement pattern despite the visual perturbation.

If movement is influenced by representational knowledge, computational strategies for a three-dimensional (3-D) object would include a description of the object derived on line from its image and compiled on the basis of a frame of reference intrinsic to the object and independent of the position of the viewer relative to the object (Marr, 1982). According to Klatzky et al. (1987), perceptual achievement of this view-independent and 3-D representation would be necessary for appropriate preshaping of the hand, earlier viewer-specific 2-D representations having failed to directly provide relevant parameters for the shaping response. The process of computing the transformation from 2-D views to a 3-D model would provide explicit motor information about "how" the hand should approach the object. In contrast, Goodale and Milner (1992) argue that the coding for action on an object includes viewer-centered computations each time the action is performed, which are largely independent of the coding used to build an object-centered picture for identification purposes.

If movement is influenced primarily by a visual input that bypasses representational systems and that is view dependent, it is predicted that the action for a 3-D apple should be different from that for a 3-D apple that is perceived as 2-D. In addition, it is predicted that visual perturbation will result in a change to the initially recruited movement pattern. In contrast, an interplay between visual pathways for action and visual pathways for representation would predict no, or little, difference in movement patterning according to perceived depth structure or with perturbation. Access to the object-specific 3-D mental model of the apple would theoretically ensure that the system "realizes" that the apple is still the same 3-D apple it has always been.

\section{EXPERIMENT 1}

Responses to perturbation of the reach-to-grasp movement can be used to infer the mechanisms underlying the planning and execution of goal-directed movements and the adaptability of the central nervous system (for review, see Haggard, 1994). Such studies have assessed a sudden shift of object position (Castiello, Paulignan, \& Jeannerod, 1991; Gentilucci, Chieffi, Scarpa, \& Castiello, 1992; Paulignan, MacKenzie, Marteniuk, \& Jeannerod, 1991) or object size (Castiello, Bennett, \& Paulignan, 1992; Castiello, Bennett, \& Stelmach, 1993; Castiello \& Jeannerod, 1991; Paulignan, Jeannerod, MacKenzie, \& Marteniuk, 1991), physical interruption to a component (Haggard \& Wing, 1995), performance of a secondary movement (Haggard, 1991), or object dimension (Castiello, Bonfiglioli, \& Bennett, 1996). 
These paradigms are used to observe how quickly the system is able to modify initial motor patterning in order to execute a different end response, and it is possible to assess the effect of perturbing one movement component both on its organization and on that of another independent but functionally coupled component. For example, the unexpected change of object size may not only disturb the organization of hand posturing (grasp component) but may oblige a response in the transport component (Castiello et al., 1993).

The aim of Experiment 1 was to assess the adaptive responses of the reach-to-grasp movement to a perturbation of perceived rather than actual object depth structure (Castiello et al., 1996). The same 3-D object was initially presented as appearing to be either 2-D or 3-D. Following a sudden change in illumination, the object presented as appearing to be 2-D would appear as 3-D. For the opposite perturbation, the object initially presented as being 3-D would suddenly appear to be 2-D.

For Experiment 1, the prediction was that if the pattern of prehension utilized in order to grasp the object is dictated by affordances, the same object should evoke different patterns according to apparent perceived structure. If this is the case, this would indicate that previous knowledge about the object or about previous grasp experiences with the same object may be overridden by on-line visual input. The strength of influence of this on-line information would be demonstrated by a switching from one to another movement pattern with a perturbation to perceived depth structure.

\section{Method}

\section{Participants}

Twenty students ( 10 women and 10 men, 23-27 years of age) volunteered to participate. All were right-handed (Edinburgh Inventory; Oldfield, 1971), reported normal or corrected-to-normal vision, and were ignorant as to the experimental purpose. Each participant attended two experimental sessions, each of approximately $1 \mathrm{~h}$ in duration.

\footnotetext{
Apparatus and Materials

The experiment was conducted under dimly lit room conditions. Details of the experimental set-up are shown in Figure 1. The participant was seated at a table that had a working surface of dimension $1 \times 1 \mathrm{~m}$. Prior to each trial, the participant placed the right hand on the table in the midsagittal plane, $15 \mathrm{~cm}$ from the thorax. In this position, the shoulder was flexed $\left(5^{\circ}-10^{\circ}\right)$, the elbow was flexed, the forearm was semipronated, and the wrist was in $10^{\circ}-15^{\circ}$ of extension. The index finger and thumb were held gently opposed, and the ulnar border of the hand rested on a pressure-sensitive starting switch.

Reflective passive markers $(0.25 \mathrm{~cm}$ in diameter) were attached to the following points of the reaching limb: (1) wrist, radial aspect of the distal styloid process of the radius; (2) index finger, radial side of the nail; (3) thumb, ulnar side of the nail; (4) middle finger, radial side of the nail; (5) ring finger, radial side of the nail; (6) little finger, radial side of the nail. Movements were recorded with the ELITE system (Ferrigno \& Pedotti, 1985), which consisted of two infrared cameras (sampling rate $100 \mathrm{~Hz}$ ) inclined at an angle of $30^{\circ}$ to the vertical and placed $1 \mathrm{~m}$ in front of and $3 \mathrm{~m}$ above the table and $2 \mathrm{~m}$ apart. The calibrated working space was a parallelepiped
}
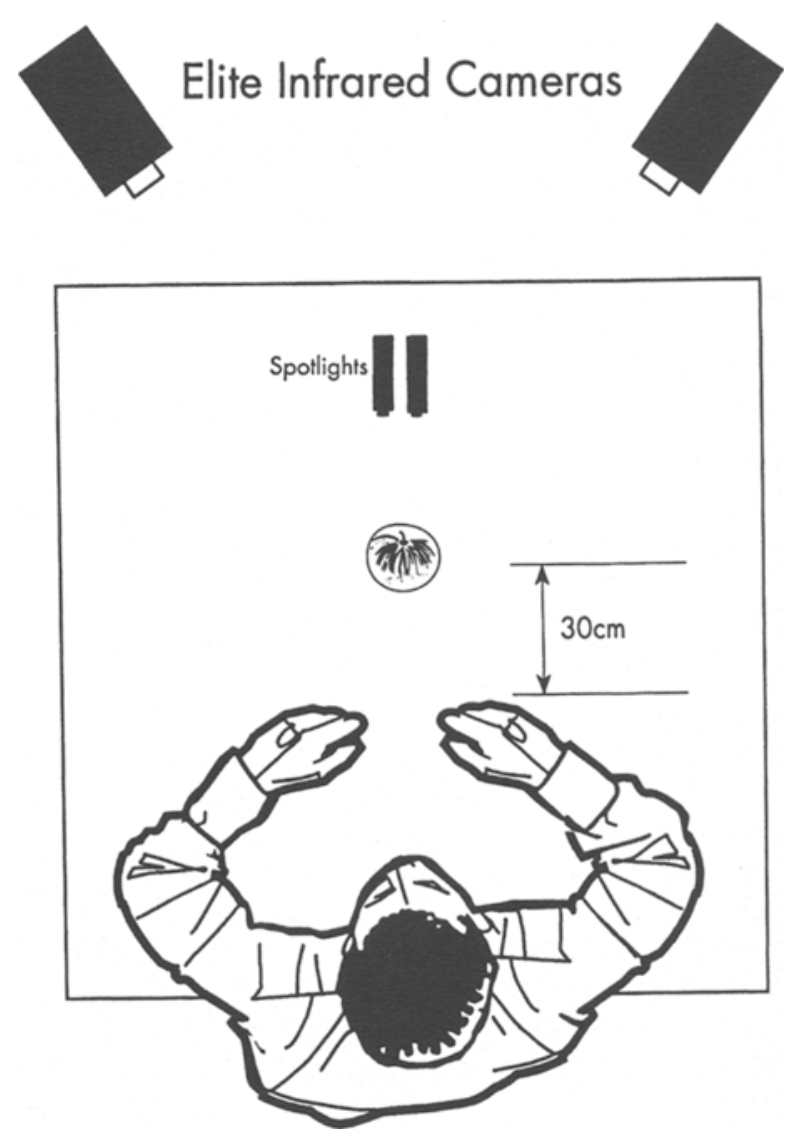

Figure 1. Experimental set-up. Note that spotlights were also positioned above the apple. Please refer to text for details. (This figure is not to scale.)

(length $=60 \mathrm{~cm}$, breadth $=30 \mathrm{~cm}$, height $=60 \mathrm{~cm}$ ) from which the spatial error measured from stationary and moving stimuli was $0.04 \mathrm{~mm}$. Coordinates of the markers, which were reconstructed with an accuracy of $1 / 3,000$ over the field of view, were sent to a host computer (PC 486). The standard deviation $(S D)$ of the reconstruction error was $1 / 3,000$ for the vertical $(y)$ axis and $1.4 / 3,000$ for the two horizontal $(x$ and $z$ ) axes.

\section{Procedure}

Experiment 1 comprised two conditions: Conditions A and B (described below).

Condition A (Perturbation from 2-D to 3-D). In Condition A, the target was an apple presented on a tray in the midsaggital plane, $30 \mathrm{~cm}$ from the switch. For the control/perturbed block, the apple appeared to be 2-D ( 80 trials). This was achieved through a darklight interplay with spotlights behind the apple. These lights did not shine directly into the participant's eyes. For 20 randomly interspersed trials, a visuodimensional perturbation was introduced at the onset of the transport component--that is, upon release of the switch. With this spotlight shift, the apple became illuminated from above so that it appeared as a 3-D object. Thus, the visual perturbation was from 2-D to 3-D.

Condition B (Perturbation from 3-D to 2-D). In Condition B, the target was an apple presented on a tray in the midsaggital plane, $30 \mathrm{~cm}$ from the switch. For the control/perturbed block, the apple appeared to be 3-D ( 80 trials). For 20 randomly interspersed trials, a visuodi- 
mensional perturbation was introduced at the onset of the transport component-that is, upon release of the switch. With this spotlight shift, the apple became illuminated from behind so that it appeared as a 2-D object. Thus, the visual perturbation was from 3-D to 2-D.

\section{Sessions}

Each participant attended two experimental sessions, one for each condition. At the beginning of the first session, two blocks of 10 trials were also performed (blocked trials): (1) the 2-D silhouette apple and (2) the 3-D apple. The order of the single block and the control/ perturbed blocks was counterbalanced across subjects.

For Condition A, the trial started with the presentation of the 2-D apple silhouette. For Condition B, the trial started with the 3-D apple in view. An auditory tone $(880 \mathrm{~Hz}$, with a duration of $250 \mathrm{msec}$, at a randomized time between 500 and $2,000 \mathrm{msec}$ after the participant had positioned the hand on the switch) signaled the participant to reach and grasp for the 2-D silhouette or the 3-D apple. To promote a "natural" movement, no instructions were given as to response speed, movement velocity, or grasp type. The participant was instructed to "grasp the object in front of you when you hear the tone" and was told that the way in which the object was illuminated might change at any moment during the movement. Each participant performed five practice trials in the same manner as the subsequent experimental block. In this group of practice trials, one perturbed trial was included. Subsequent analysis of these practice trials gave the same results as found for the experimental trials. No formal assessment of the participants' subjective perceptions was undertaken.

\section{Data Processing}

The ELIGRASP processing package (B|T|S|, 1994) was used to assess the data. This gave a 3-D reconstruction of the marker posit1ons. The data were then filtered using a FIR linear filter-transition band of $1 \mathrm{~Hz}$ (sharpening factor = 2; D'Amico \& Ferrigno, $1990,1992)$. The transport component was assessed by analyzing the trajectory, velocity, and acceleration profiles of the wrist marker. The manipulation component was assessed by analyzing the trajectory of each of the hand markers and the distance between the markers positioned on the index finger and thumb. The velocity of the opening and closing action of the digits was also assessed. "Movement initiation time" (so called because no emphasis was placed on a rapid response) was taken from release of the starting switch. The end of the movement was taken as the time when the fingers closed upon the fruit and there was no further change in the distance between the index finger and thumb. The period following this, whereby the fruit was brought to the starting position, was not assessed. The dependent variables were (1) initiation time; (2) movement duration; (3) for the transport component, the times to peak velocity, peak acceleration, and peak deceleration of the wrist marker, and the amplitudes of these peaks (amplitude peak velocity, amplitude peak acceleration, and amplitude peak deceleration); (4) for the manipulation component, the times to peak grip aperture and to peak grip velocity, the amplitude of the aperture and velocity peaks, and the time to specification of the index finger for precision grip (specification time). This latter parameter refers to the time at which the index finger deviates from the more ulnar digits as it prepares for the precision grip (break detection algorithm; Castiello et al., 1993).

\section{Results and Discussion}

\section{Blocked Trials}

In this section, results for the blocked trials are reported. Qualitatively, the results were clear. The 2-D apple was almost always grasped as if it were a disk (170 of 200 trials) - that is, with a large precision grip between the index finger and thumb (Figures $2 \mathrm{~B}$ and $2 \mathrm{C}$ ). In contrast, the 3-D apple was almost always grasped with a whole-hand prehension (180 of 200 trials; Figure $2 \mathrm{~A}$ ), whereby all digits and some part of the palm contacted its surface. For the large precision grip, specification time occurred at an average of $200 \mathrm{msec}(S D=35)$ after switch release. (Note that two methods were used to "score" the type of grasp used. During the experiment, a researcher viewed the task performance and noted the type of grasp as being precision grip, whole-hand prehension, fingertip, or unknown. Following data capture and initial analysis, the trajectories of the digits were viewed to describe the type of grasp that was employed.)

Interestingly, only grasp type changed according to perceived object depth structure. Movement duration and kinematics of both the transport and the manipulations component were remarkably similar in both the temporal domain and the spatial domain when comparing 2-D with 3-D trials. With regard to the wrist marker, peak acceleration, peak velocity, and peak deceleration of the 2-D reaching action occurred at similar times as peak acceleration, peak velocity, and peak deceleration of the action to the 3-D apple (see Table 1). With regard to the thumb and index finger markers, the time of maximum grip aperture and the amplitude of this aperture were not significantly different when comparing the two conditions.

\section{Condition A}

Results for Condition A are summarized in Table 2.

Control trials. Qualitatively, the results for control trials that were interspersed with perturbed trials were similar to those for blocked trials. Again, the majority of trials to the object that was perceived as 2-D were performed with a large precision grip involving only the thumb and index finger ( $n=1,312 ; 82 \%)$ or also including the middle finger $(n=227 ; 14.2 \%)$. Of those involving only the index finger and thumb, most $(90 \%)$ were performed with the ulnar fingers flexed out of the way (Figure 2B), with specification for precision grip (i.e., the time at which the index finger deviated away from the more ulnar digits) occurring at an average time of $198 \mathrm{msec}(S D=36)$ after switch release. Only 61 cases (3.8\%) of all trials to the perceived 2-D apple were performed using a whole-hand

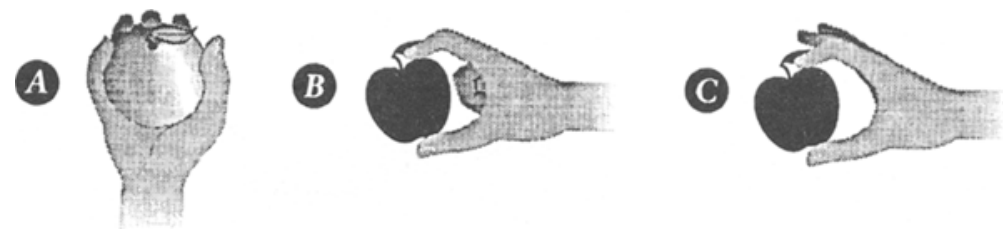

Figure 2. (A) Typical whole-hand prehension grasp of the 3-D apple. (B) Large precision grip of the 2-D apple, ulnar fingers flexed. (C) Large precision grip of the 2-D apple, ulnar fingers extended. 
Table 1

Mean Values of Movement Durations and Kinematic Parameters for the Blocked Trials of Experiments 1 and 2

\begin{tabular}{|c|c|c|c|c|c|c|c|c|}
\hline & \multicolumn{8}{|c|}{ Blocked Trials } \\
\hline & \multicolumn{4}{|c|}{ Experiment 1} & \multicolumn{4}{|c|}{ Experiment 2} \\
\hline & \multicolumn{2}{|c|}{$2-\mathrm{D}$} & \multicolumn{2}{|c|}{ 3-D } & \multicolumn{2}{|c|}{$2-\mathrm{D}$} & \multicolumn{2}{|c|}{ 3-D } \\
\hline & $M$ & $S D$ & $M$ & $S D$ & $M$ & $S D$ & $M$ & $S D$ \\
\hline Movement Duration (msec) & 737 & 74 & 745 & 83 & 728 & 74 & 741 & 76 \\
\hline \multicolumn{9}{|l|}{ Transport Component } \\
\hline Time to peak acceleration (msec) & 220 & 36 & 221 & 38 & 220 & 34 & 219 & \\
\hline Time to peak velocity (msec) & 303 & 34 & 312 & 37 & 307 & 38 & 319 & 40 \\
\hline Time to peak deceleration (msec) & 401 & 45 & 421 & 39 & 418 & 48 & 432 & 45 \\
\hline \multicolumn{9}{|l|}{ Manipulation Component } \\
\hline Amplitude maximum grip aperture (mm) & 108 & 2 & 107 & 3 & 108 & 4 & 110 & \\
\hline Time to maximum grip aperture (msec) & 427 & 44 & 436 & 47 & 444 & 39 & 453 & 46 \\
\hline Specification time (msec) & 200 & 35 & & & 204 & 31 & & - \\
\hline
\end{tabular}

prehension. Almost all trials (92\%) to the perceived 3-D apple were performed as a whole-hand prehension.

Remarkably, a precision grip for the 2-D apple was utilized even for most $(80 \%)$ of the control trials that were performed immediately after the perturbed 3-D trials where a whole-hand prehension had been utilized.

Kinematic results for the control trials mirrored those obtained for the blocked trials. When comparing blocked trials with 10 randomly chosen control trials with the apparently 2-D apple, no significant differences were found.

Perturbed trials. Analysis of the perturbed trials showed that each participant began the movement with a large precision grip but then rapidly changed the output to a whole-hand prehension. Mirroring the results of the control trials, specification for precision grip occurred at a mean of $193 \mathrm{msec}(S D=30)$ after switch release, indicating initial programming for precision grip in response to the apparently 2-D apple (Figure $3 \mathrm{~A}$ ). An obvious halting of this output was revealed by a dip in the grip opening velocity profile at a mean of $298 \mathrm{msec}(S D=$ 32). Changes in the trajectories of the ulnar digits (middle, ring, little) showed that whole-hand prehension was then recruited (Figure $3 \mathrm{C}$ ) at an average of $342 \mathrm{msec}$ after switch release (and thus after perturbation). Maximum grip aperture for the perturbed trials showed no significant difference from that for control trials-an expected result, given that the diameter of the object remained constant.

The transport component showed an even earlier recognition of the perturbation. The time to peak wrist acceleration was approximately $60 \mathrm{msec}$ earlier for perturbed trials than for control trials [ 161 vs. $218 \mathrm{msec} ; F(1,19)=$ $22.06, p<.0001$; see Figure 4; Condition A]. Another significant difference between the perturbed and control trials was that movement duration was longer for the former [801 vs. $743 \mathrm{msec} ; F(1,19)=27.05, p<.0001]$.

\section{Condition B}

Results for Condition B are summarized in Table 2.

Control trials. For this opposite perturbation, a wholehand prehension for the 3-D apple was utilized for most (320 of 400) of the control trials that were performed immediately after the perturbed 3-D to 2-D trials, where a precision grip had been utilized. For the remaining control trials $(1,200)$, almost all $(88 \%)$ were performed in the same manner. However, for 224 (18\%) of the control tri-

Table 2

Mean Values of Relevant Dependent Measures of Experiment 1

\begin{tabular}{|c|c|c|c|c|c|c|c|c|}
\hline & \multicolumn{4}{|c|}{ Condition A } & \multicolumn{4}{|c|}{ Condition B } \\
\hline & \multirow{2}{*}{\multicolumn{2}{|c|}{$\frac{\text { Control Trials }}{2-\mathrm{D}}$}} & \multirow{2}{*}{\multicolumn{2}{|c|}{$\frac{\text { Perturbed Trials }}{2-D \rightarrow 3-D}$}} & \multirow{2}{*}{\multicolumn{2}{|c|}{$\frac{\text { Control Trials }}{3-\mathrm{D}}$}} & \multirow{2}{*}{\multicolumn{2}{|c|}{$\frac{\text { Perturbed Trials }}{3-\mathrm{D} \rightarrow 2-\mathrm{D}}$}} \\
\hline & & & & & & & & \\
\hline & $M$ & $S D$ & $M$ & $S D$ & $M$ & $S D$ & $M$ & $S D$ \\
\hline Movement Duration (msec) & 743 & 80 & 801 & 85 & 736 & 89 & 787 & 85 \\
\hline \multicolumn{9}{|l|}{ Transport Component } \\
\hline Time to peak acceleration (msec) & 218 & 38 & 161 & 28 & 225 & 38 & 228 & 35 \\
\hline Time to peak velocity (msec) & 298 & 43 & 257 & 38 & 312 & 36 & 316 & 35 \\
\hline Time to peak deceleration (msec) & 445 & 56 & 439 & 43 & 456 & 51 & 448 & 54 \\
\hline \multicolumn{9}{|l|}{ Manipulation Component } \\
\hline Amplitude maximum grip aperture $(\mathrm{mm})$ & 109 & 3 & 110 & 2 & 108 & 2 & 108 & 3 \\
\hline Time to maximum grip aperture (msec) & 465 & 54 & 454 & 48 & 469 & 53 & 471 & 50 \\
\hline Specification time (msec) & 198 & 36 & 193 & 30 & \multicolumn{2}{|c|}{-} & 214 & 32 \\
\hline
\end{tabular}

Note-2-D, apple perceived as two dimensional; $3-\mathrm{D}$, apple perceived as three dimensional; $2-\mathrm{D} \rightarrow 3-\mathrm{D}$, visual perturbation from a $2-D$ to a $3-D$ visual perception; $3-D \rightarrow 2-D$, visual perturbation from a $3-D$ to a $2-D$ visual perception. 
A

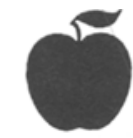

B
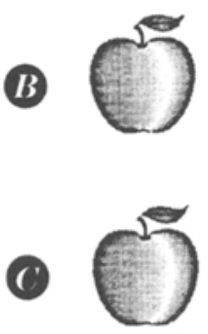

(D)

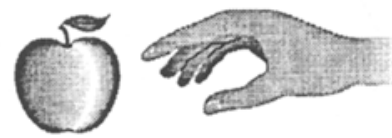

(E)

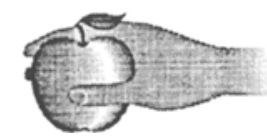

Figure 3. Sequence of hand postures during a perturbed trial where, at movement onset to the apparently 2-D apple, the lighting changed so that the apple appeared to be 3-D. The apple is shown as it appeared to the subject. (A) Starting position. (B) Spotlighting changed so that the apple appeared to be 3-D. The index finger and thumb show specification for precision grip in response to the initial 2-D presentation. (C) Ulnar fingers show recruitment for whole-hand prehension in response to the 3-D presentation. (D) Hand posture toward completion of the movement. (E) Whole-hand prehension grasp of the apple.

als, the participants reached for the 2-D apple and grasped it by positioning the tips of their fingers all around its contour. However, this latter "fingertip" grasp was clearly different from the whole-hand prehension used for the 3-D apple, where contact by all the digits and the palm was evident. When comparing blocked trials with 10 randomly chosen control trials to the apparently 3-D apple, no differences in movement kinematics were found.

Perturbed trials. Analysis of the perturbed trials showed that the participant began with a whole-hand prehension but then rapidly changed the output to a precision grip. In very few cases $(2 \%)$, the perturbed trial ended with a fingertip grasp. Changes in the trajectories of the ulnar digits (middle, ring, little) showed that the digits began to no longer travel toward grasp participation at an average of $291 \mathrm{msec}(S D=37)$ after switch release (and thus after perturbation). Unlike the opposite perturbation, the transport component did not show early signs of recognition of the perturbation. The time to peak wrist acceleration was similar for perturbed and control trials [ 228 vs. $225 \mathrm{msec} ; F(1,19)=1.04, p>.05$; see Figure 4; Condition B]. However, and like the 2-D to 3-D perturbation, the parameter of movement duration showed a significant difference between the perturbed and control trials, being of greater duration for the former [787 vs. $736 \mathrm{msec} ; F(1,19)=9.03, p<.001]$.

Overall, the results for the two types of perturbation indicate that the motor patterns we use in interacting with an object are strongly influenced by the way we perceive the dimensions, or depth structure, of an object in real time. Furthermore, it appears that the passage from a 3-D to a 2-D representation for motor output is smoother and easier than the passage from a 2-D to a 3-D representation. In the former case, minimal modifications to the transport component are necessary in order to promote the motor output change (Figure 4; Condition B). The passage from a 2-D to a 3-D representation and the corre-

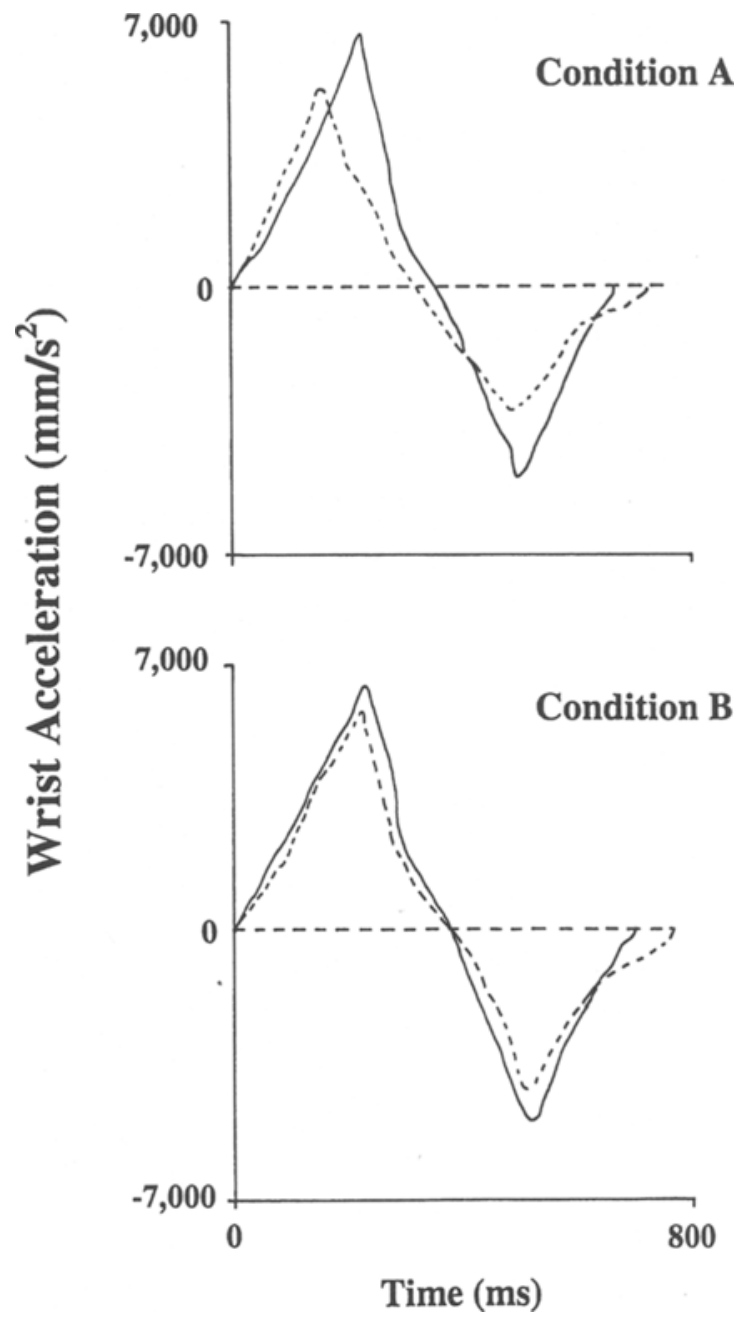

Figure 4. An example of the acceleration profiles for a control 2-D trial (solid line) and a perturbed trial (dotted line) of 1 subject for Condition A (2-D to 3-D perturbation) and Condition B (3-D to 2-D perturbation). Note that the peak of acceleration for the perturbed trials was earlier than that of the control trials only for Condition $\mathbf{A}$. 
sponding shift in motor output appear to be more demanding from a computational point of view. The reason for suggesting this difference in ease of "dimensional" transition stems largely from the results for the transport component. With the more difficult transition from 2-D to 3-D, the motor system compensates rapidly by anticipating the time of peak acceleration (Figure 4; Condition A). This is presumably to allow greater time for the final stages of the action, as supported by the noticeable increase in movement duration for these 2-D to 3-D perturbed trials. In contrast, the transport component of the 3-D to 2-D perturbation shows no obvious indication of compensation except for an increase in the duration of movement (Figure 4; Condition $\mathrm{B}$ ).

\section{EXPERIMENT 2}

Results of Experiment 1 point to the importance of online visual perception for the implementation of a motor pattern. However, the experimental design of Experiment 1 did not include "catch trials," whereby in Condition A, for example, the perturbed trials did not correspond to a shift from a 2-D to a 3-D perceived view but from a 2-D perceived view to a 2-D picture of an apple. The reason for including catch trials is to tease out effects that are due to particular features of the experimental paradigm from those that could be attributed to the perceived depth structure of the object. As an example, it could be argued that the backlighting made the apple appear to be less distinct or blurred about its edges, and this visual characteristic, rather than perceived depth structure, determined the results. Other factors might include expectancy effects, changes in illumination, or changes in color (i.e., a black silhouette to a red apple).

In Condition A of Experiment 2, the catch trials consisted of a change in illumination from backlighting to above lighting of a cardboard figure of an apple (i.e., 2$D$ to 2-D perturbation), which was done in order to determine whether the results for the 2-D to 3-D perturbed trials for the real apple in Experiment 1 could be attributed to a shift in illumination or to a shift in perceived depth structure. If the former were true, the expectation would be that the 2-D to 2-D trials would not differ from the 2-D to 3-D trials. A similar result would be predicted if the initial "blurriness" of the image determined the response to perturbation. In Condition B of Experiment 2, illumination of the real apple was interrupted for a brief moment to mimic the sudden "on-off" shift that was characteristic of the perturbed trials in Experiment 1. If it were this particular lighting characteristic of the paradigm that determined the results, the expectation again would be that the 3-D to 3-D perturbation (i.e., the onoff condition) would show results similar to those of the 3-D to 2-D perturbation. Given the hypothesis that the perceived on-line information of an object's dimensional features determines the characteristics of an action directed to that object, it was predicted that the results for the catch perturbed trials would differ from the results for the perturbed trials, where a shift in perceived depth structure was intended. The findings of no differences between Experiment 2 and Experiment 1 for the real perturbed as opposed to the catch perturbed trials would add further support to the contention that perceived depth structure plays an important role for motor output formation.

\section{METHOD}

\section{Participants}

Twenty students ( 10 women and 10 men, $21-25$ years of age) participated in this experiment. None had participated in Experiment 1 , but they had the same characteristics as the participants of the previous experiment.

\section{Apparatus and Materials}

Experiment 2 was conducted in a darkened room, such that, when seated at the table, the participant could not see the table surface or objects positioned on the table. Apart from this difference in the ambient room lighting, the apparatus was the same as that utilized in Experiment 1, except that, for Condition A, an additional target stimulus - a picture of a real apple mounted on a piece of appleshaped cardboard-could be employed. This cardboard apple was attached to an L-shaped support consisting of a horizontal bar and a vertical bar. The support did not limit performance of either a whole-hand prehension or a large precision grip.

\section{Procedure}

As in Experiment 1, Experiment 2 comprised two conditions, one for each of two experimental sessions. For both of these sessions, the participant was instructed to close the eyes following completion of each trial. During each of these periods of eye closure, the experimenter changed, or pretended to change, the stimulus. In this way, the cardboard stimulus could be positioned without knowledge of the depth structure of the stimulus prior to the onset of each trial. In this eye-closure period, factors such as the amount of noise made by the experimenters in changing the stimulus and the time taken between each trial were kept constant across different trial types.

Condition A (Perturbation from 2-D to 3-D). The majority of trials $(n=80)$ in Condition A were control trials to the perceived 2-D apple (i.e., the apple was illuminated from behind). For $10 \%$ $(n=10)$ of the total number of trials, the real apple was replaced with the cardboard 2-D apple during the period of eye closure. This cardboard shape was illuminated from behind; because its diameter and shape were the same as that of the real apple, its black silhouette image was the same as that of the real apple. Upon eye opening, the trial commenced with the participant moving to grasp the stimulus; however, with release of the starting switch, the spotlight shifted to illuminate the shape from above (perturbed 2-D to 2-D catch trials). For $10 \%$ of the trials, the perturbation was the same as for Condition A of Experiment 1 -that is, the real apple was initially illuminated from behind (perceived 2-D apple), with the shift of illumination to the spotlight positioned above (3-D apple). These perturbed trials were randomly interspersed among the control trials.

Condition B (Perturbation from 3-D to 2-D). For the 80 control trials in Condition B, the 3-D apple was presented (i.e., illuminated from above). Ten catch trials were presented by initially illuminating the real apple from above and then quickly turning this same spotlight off (less than $50 \mathrm{msec}$ ) and on again at switch release (perturbed 3-D to 3-D catch trials). For a further $10 \%$ of trials, the perturbation was the same as for Condition B of Experiment 1 that is, a 3-D apple was presented initially, with a shift of illumination to the spotlight behind the real apple. Again, perturbed trials were randomly interspersed among control trials. 
Table 3

Mean Values of Relevant Dependent Measures of Experiment 2

\begin{tabular}{|c|c|c|c|c|c|c|c|c|}
\hline & \multicolumn{4}{|c|}{ Condition A } & \multicolumn{4}{|c|}{ Condition B } \\
\hline & \multirow{2}{*}{\multicolumn{2}{|c|}{$\frac{\text { Control Trials }}{2-\mathrm{D}}$}} & \multirow{2}{*}{\multicolumn{2}{|c|}{$\frac{\text { Perturbed Trials }}{2-\mathrm{D} \rightarrow 3-\mathrm{D}}$}} & \multirow{2}{*}{\multicolumn{2}{|c|}{$\frac{\text { Control Trials }}{\text { 3-D }}$}} & \multirow{2}{*}{\multicolumn{2}{|c|}{$\begin{array}{c}\text { Perturbed Trials } \\
3-\mathrm{D} \rightarrow 2-\mathrm{D}\end{array}$}} \\
\hline & & & & & & & & \\
\hline & $M$ & $S D$ & $M$ & $S D$ & $M$ & $S D$ & $M$ & $S D$ \\
\hline Movement Duration (msec) & 698 & 78 & 756 & 80 & 701 & 76 & 774 & 81 \\
\hline $\begin{array}{l}\text { Transport Component } \\
\text { Time to peak acceleration (msec) } \\
\text { Time to peak velocity }(\mathrm{msec}) \\
\text { Time to peak deceleration }(\mathrm{msec})\end{array}$ & $\begin{array}{l}244 \\
356 \\
476\end{array}$ & $\begin{array}{l}30 \\
44 \\
51\end{array}$ & $\begin{array}{l}189 \\
299 \\
467\end{array}$ & $\begin{array}{l}25 \\
32 \\
48\end{array}$ & $\begin{array}{l}237 \\
345 \\
478\end{array}$ & $\begin{array}{l}31 \\
34 \\
54\end{array}$ & $\begin{array}{l}241 \\
346 \\
481\end{array}$ & $\begin{array}{l}37 \\
41 \\
53\end{array}$ \\
\hline $\begin{array}{l}\text { Manipulation Component } \\
\text { Amplitude maximum grip aperture }(\mathrm{mm}) \\
\text { Time to maximum grip aperture }(\mathrm{msec}) \\
\text { Specification time (msec) }\end{array}$ & $\begin{array}{l}109 \\
498 \\
201\end{array}$ & $\begin{array}{r}3 \\
53 \\
23\end{array}$ & $\begin{array}{l}109 \\
503 \\
200\end{array}$ & $\begin{array}{r}3 \\
51 \\
21\end{array}$ & $\begin{array}{l}108 \\
500\end{array}$ & $\begin{array}{r}2 \\
48\end{array}$ & $\begin{array}{l}110 \\
500 \\
210\end{array}$ & $\begin{array}{r}3 \\
50 \\
25\end{array}$ \\
\hline
\end{tabular}

Note- -2-D, apple perceived as two dimensional; 3-D, apple perceived as three dimensional; 2-D $\rightarrow 3-\mathrm{D}$, visual perturbation from a 2-D to a 3-D visual perception; 3-D $\rightarrow 2-\mathrm{D}$, visual perturbation from a 3-D to a 2-D visual perception.

In all other respects, the procedure was the same as that utilized for Experiment 1.

\section{Results and Discussion}

Values are presented in Table 3. Results for the catch trials confirmed the importance of the on-line interpretation of an object dimension for motor output. For the perturbations from the silhouette to the 2-D picture of an apple (i.e., the 2-D to 2-D catch perturbation), the subjects did not change the initially executed precision type grasp to a whole-hand prehension. The kinematic para-

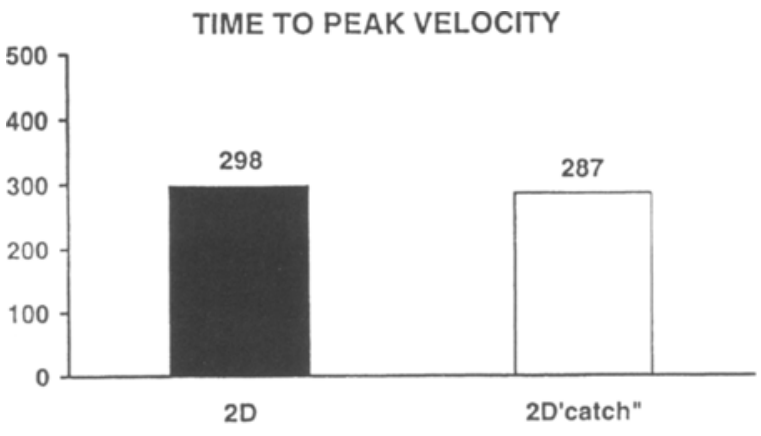

TIME TO PEAK DECELERATION

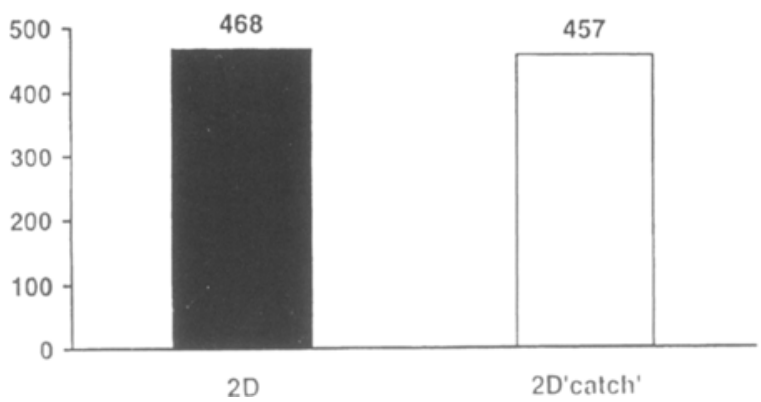

meterization for these catch trials mirrored that of the control trials. As an example, for the transport component peak acceleration, peak deceleration and peak velocity occurred at similar times when comparing the catch trials with the control trials (see Figure 4). For the manipulation component, time to maximum grip aperture and specification time were also similar (see Figure 5). These results indicated that a simple shift of illumination could not account for the results observed for the perturbed trials of Experiment 1 . In addition, given that the cardboard shape and the real apple had the same sil-

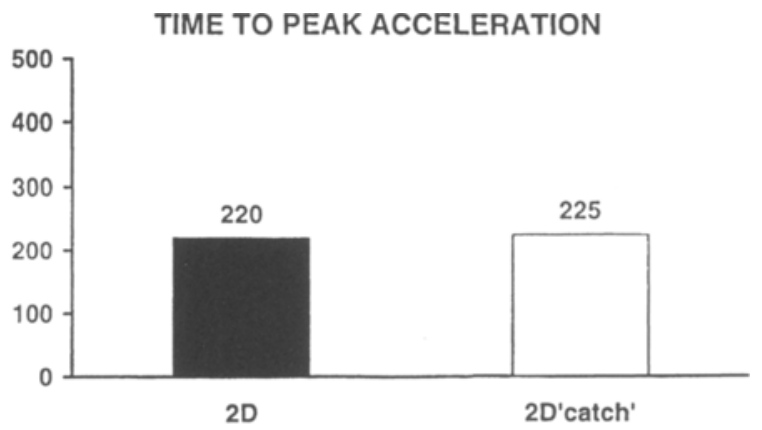

TIME TO MAXIMUM GRIP APERTURE

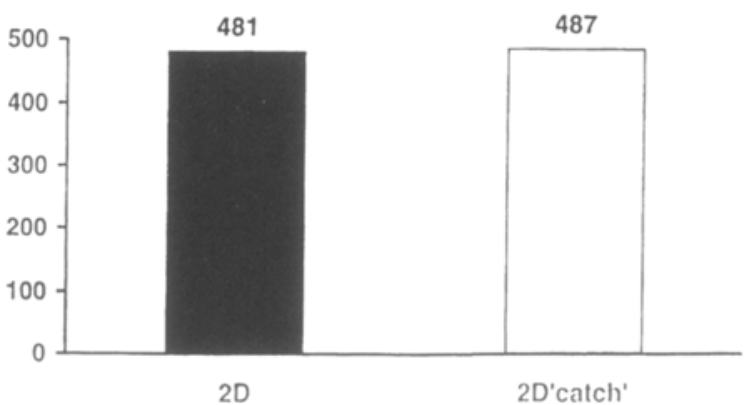

Figure 5. Comparison between kinematic values obtained for the 2-D control and 2-D catch trials of Experiment 2. Comparison analyses showed no significant differences between these two types of trial for the parameters illustrated in this diagram. 
houette at trial onset, loss of information about the precise shape of the 3-D as a result of illumination from behind probably cannot explain the results of Experiment 1 .

For the catch perturbation from the real 3-D apple to the real 3-D apple, the initially recruited whole-hand prehension showed no signs of disruption. This indicated that a change in illumination (from on to off to on) also could not account for the results of Experiment 1.

In all other respects, the results mirrored those of Experiment 1. For Condition A (2-D to 3-D), the time to peak acceleration was earlier for real perturbed trials than for control trials $[189$ vs. $244 \mathrm{msec}, F(1,19)=10.32, p<$ .001 ], whereas for Condition B trials (3-D to 2-D), peak acceleration occurred at the same time for control and perturbed trials ( 237 and $241 \mathrm{msec}$, respectively). For Conditions $\mathrm{A}$ and $\mathrm{B}$, movement duration was longer for real perturbed trials than for control trials [Condition A, 756 vs. $698 \mathrm{msec}, F(1,19)=33.05, p<.0001$; Condition $\mathrm{B}, 774$ vs. $701 \mathrm{msec}, F(1,19)=27.32, p<.0001]$. For the manipulation component, specification time occurred at 201 and $200 \mathrm{msec}$ for control and perturbed trials, respectively. Given that the times to peak acceleration in both perturbed and control trials appeared to be longer in Experiment 2 than in Experiment 1, and that the inverse pattern was apparent for movement duration, comparison analyses were conducted. No differences were found. This suggested that the differences in ambient room lighting did not lead to differences in results.

\section{GENERAL DISCUSSION}

This study assessed the effects of the perceived depth structure of an object on the patterning of a prehension movement involving that object. For Experiment 1, nothing about the physical characteristics of the object, an apple, changed. The only experimental manipulation was the way in which the dimensions of the apple were assumed to have been perceived: 2-D or 3-D. If an apple was perceived as being $2-D$, the participants usually utilized a large precision grip between the index finger and thumb. If the apple was perceived as being 3-D, wholehand prehension involving all the digits and palmar contact was utilized. On their own, these results suggest that visual mechanisms for interpreting an object's dimensions directly influence motor selection pathways, without, necessarily, access to a 3-D central nervous system representation of the object.

The strength of the effects of this on-line visual interpretation was confirmed with the perturbation results. For the 2-D to 3-D perturbed trials, the participant initially perceived the apple as 2-D. Upon initiating the reach to the apple, it suddenly "became" 3-D. The initially recruited 2-D pattern of a large precision grip was curbed, and a whole-hand prehension was used to grasp the apple. This rapid modification was evident in both the manipulation component and the transport component of the action, and it is consistent with other perturbation study results (Castiello et al., 1991; Paulignan, Jeannerod, et al., 1991; Paulignan, MacKenzie, et al., 1991). For example, and as previously found with paradigms where object position was perturbed, in the perturbation from 2-D to 3-D, the transport component showed an earlier arm peak acceleration for perturbed trials than for control trials, indicating recognition of the change in position in order to facilitate changes in the manipulation component. The changes in the manipulation component are consistent with an arrest of the precision grip with the subsequent recruitment of the 3-D whole-hand prehension pattern. Intuitively, this change seems unnecessary, given that the apple is still the same apple. However, it demonstrates the potent effect of the interpretation of an object's dimensions in commandeering a defined motor output.

For the 3-D to 2-D perturbed trials, the apple suddenly "became" 2-D as the participant initiated the reaching action. The results indicated that the initially recruited 3-D motor pattern changed to a large precision grip or to a fingertip grasp. However, in the case of this perturbation, modification was only to the manipulation component rather than to the transport component. This lack of transport modification with depth structure perturbation from 3-D to 2-D suggests that the movement organization for the 3-D object had sufficient flexibility for use as a 2-D motor pattern. This was not the case for the opposite perturbation. In a change from 2-D to 3-D presentation, the initially activated transport component parameterization was not sufficient for use in a 3-D pattern. This recognition and the subsequent changes to the transport component appears to occur at a very early stage of the visuomotor translation. Unlike the findings in size perturbations where peak deceleration-a relatively late parameter of the transport component-is anticipated (Castiello et al., 1993; see also Paulignan, MacKenzie, et al., 1991), a 2-D to 3-D depth structure perturbation results in changes to peak acceleration-a very early temporal parameter of the transport component. This early transport response is reminiscent of the results found with perturbation of object location where peak acceleration is anticipated when the spatial location of a target to reach is manipulated (Castiello et al., 1991; Paulignan, Jeannerod, et al., 1991; Paulignan, MacKenzie, et al., 1991). With both the "depth structure" perturbation and the "location" perturbation, the earliest response was in the transport component, suggesting an important spatial element in the visuomotor processing of dimension.

Do these results demonstrate that a stored model of the object has been utilized somewhere in the process between visual interpretation of its dimensions and the motor output? After all, an apple is a familiar object-one that is often grasped, and one that could be presumed to have a central nervous system representation. From the results of the present study, it can be assumed that even if we recognize that the silhouette is an apple, and even if we have previously grasped that exact same apple when it was perceived as 3-D, we do not appear to scan automat- 
ically a canonical mental dictionary of object models that, presumably, would suggest to utilize a whole-hand prehension. Alternatively, it may be that we scan this model, but that its influence is overridden by the powerful effects of on-line visual interpretation of the perceived depth structure.

Because the motor outputs can differ according to perceived depth structure, the results from the present study support the idea of stages, or different channels, of visual interpretation. A single-level representation of an object is not supported, because this would predict identical outputs to the same object, irrespective of whether it was perceived as 2-D or 3-D. Similarly, the results do not support the idea that presentation of a normally perceived 3-D object in a novel 2-D form elicits a matching process with the already stored views of the 3-D object. Adopting Marr's (1982) model, it would appear that, under certain conditions, a visual stage related to the composition of $2-D$ to $2 \frac{1}{2}-\mathrm{D}$ attributes accesses motor outputs using channels that bypass, or pass through but ignore, a stage related to the composition of 3-D attributes. The very low incidence of trials where a whole-hand prehension is utilized for the 2-D apple could, however, indicate a default level of interchannel cross talk or indicate that a 3-D representation is not entirely ignored. The presence of "hybrid" grasps-for example, those that are somewhere between a large precision grip and a whole-hand prehension-further suggests a certain level of motor output/representational cross talk.

It is concluded that motor computational processes can be strongly influenced by what is perceived about the object and that this perception can override the influence exerted by existing representations (what we know about the object) and by existing visuomotor neural coding (what we have previously enacted upon and experienced about the object). Hypothetically, this points to the existence of an object properties system where only attributes that are relevant for movement organization (e.g., size, shape, dimension, etc.) are considered for motor output computation. Using the terminology of Goodale and Milner (1992), this could be operationalized through "how" visuomotor channels. Jeannerod (1994) proposed that these channels subserve "functionality" and "affordance" (Gibson, 1969), and they complement, at least theoretically, the "what" ventral object recognition channel passing through temporal cortex and the "where" dorsal object-localization channel passing through the parietal cortex (Ungerleider \& Mishkin, 1982). The results of this study contribute to an understanding of the operationalization of the "how" channel. For example, given that the rapid response to the depth structure perturbation is very similar to that observed when object position is varied, object dimension could be specified at the same level as location and orientation. By passing through these channels, the object may obtain a parallel level of spatial mapping for object recognition such as a "geometric mapping." In other words, it is suggested that the results of this study provide further evidence for a distinction between the "what" and "how" pathways (see Goodale \& Milner, 1992). The "how" pathway for processing dimension has early access to spatial pathways, and to presemantic components/neural mechanisms that bypass elements of the "what" channel.

\section{REFERENCES}

Aglioti, S., DeSouza, J. F. X., \& Goodale, M. A. (1995). Size-contrast illusions deceive the eye but not the hand. Current Biology, 5, 679-685.

Bennett, K. M. B., \& Castiello, U. (Eds.) (1994). Insights into the reach to grasp movement. Amsterdam: Elsevier.

BRENNER, E., \& SMEETS, J. B. J. (1996). Size illusion influences how we lift but not how we grasp an object. Experimental Brain Research, 111 473-476.

CASTIELLO, U. (1996). Grasping a fruit: Selection for action. Journal of Experimental Psychology-Human Perception \& Performance, 22, 582-603.

Castiello, U., Bennett, K. M. B., \& Paulignan, Y. (1992). Does the type of prehension influence the kinematics of reaching? Behavioural Brain Research, 50, 7-15.

Castiello, U., Bennett, K. M. B., \& Stelmach, G. E. (1993). Reach to grasp: The natural response to a perturbation of object size. Experimental Brain Research, 94, 165-178.

Castiello, U., Bonfiglioli, C., \& Bennett, K. M. B. (1996). How perceived object dimension influences prehension. NeuroReport, 7 , 825-829.

Castiello, U., \& Jeannerod, M. (1991). Measuring time to awareness. NeuroReport, 2, 797-800.

Castiello, U., Paulignan, Y., \& Jeannerod, M. (1991). Temporal dissociation of motor responses and subjective awareness. Brain, 114, 2639-2655

D'Amico, M., \& Ferrigno, G. (1990). Technique for the evaluation of derivatives from noisy biomechanical displacement data using a model-based bandwidth-selection procedure. IEEE Transactions on Biomedical Engineering, 28, 407-415.

D'Amico, M., \& Ferrigno, G. (1992). Comparison between the more recent techniques for smoothing and derivative assessment in biomechanics. IEEE Transactions on Biomedical Engineering, 30, 193-204.

Ferrigno, G., \& PeDotti, A. (1985). ELITE: A digital dedicated hardware system for movement analysis via real-time TV signal processing. IEEE Transactions on Biomedical Engineering, 32, 943-950.

Gentilucci, M., Chieffi, S., Scarpa, M., \& Castiello, U. (1992). Temporal coupling between transport and grasp components during prehension movements: Effects of visual perturbation. Behavioural Brain Research, 47, 71-82.

GiBSON, E. J. (1969). Perceptual learning and development. New York: Appleton-Century-Crofts.

Goodale, M. A., \& Milner, A. D. (1992). Separate visual pathways for perception and action. Trends in Neurosciences, 15, 20-25.

Goodale, M. A., Mil.ner, A. D., Jakobson, L. S., \& Carey, D. P. (1991). A neurological dissociation between perceiving objects and grasping them. Nature, 349, 154-156.

HAGGARD, P. (1991). Task coordination in human prehension. Journal of Motor Behaviour, 23, 25-37.

HaGgard, P. (1994). Perturbation studies of coordinated prehension. In K. M. B. Bennett \& U. Castiello (Eds.), Insights into the reach to grasp movement (pp. 151-170). Amsterdam: North-Holland.

Haggard, P., \& WING, A. (1995). Coordinated responses following mechanical perturbation of the arm during prehension. Experimental Brain Research, 102, 483-494.

Jakobson, L. S., Archibald, Y. M., Carey, D. P., \& Goodale, M. A. (1991). A kinematic analysis of reaching and grasping movements in a patient recovering from optic ataxı. Neuropsychologia, 29, 803-809.

JEANNEROD, M. (1981). Intersegmental coordination during reaching at natural visual objects. In J. Long \& A. Baddeley (Eds.), Attention and performance $I X$ (pp. 153-169). Hillsdale, NJ: Erlbaum. 
JeANNERoD, M. (1994). Object oriented action. In K. M. B. Bennett \& U. Castiello (Eds.), Insights into the reach to grasp movement (pp. 3-15). Amsterdam: North-Holland.

Klatzky, R. L., McCloskey, B. P., Doherty, S., Pellegrino, J., \& SMITH, T. (1987). Knowledge about hand shaping and knowledge about objects. Journal of Motor Behaviour, 19, 197-213.

MARR, D. (1982). Vision. San Francisco: W. H. Freeman.

Milner, A. D., Perrett, D. I., Johnston, R. S., Benson, P. J., Jordan, T. R., Heeley, D. W., Bettucci, D., Mortara, F., Mutani, R., Terazzi, E.. \& Davidson, D. L. W. (1991). Perception and action in "visual form agnosia." Brain, 114, 405-428.

OLDFIELD, R. C. (1971). The assessment and analysis of handedness: The Edinburgh inventory. Neuropsychologia, 9, 97-113.

Paulignan, Y., Jeannerod, M., MacKenzie, C., \& Marteniuk, R.
(1991). Selective perturbation of visual input during prehension movements: 2 . The effects of changing object size. Experimental Brain Research, 87, 407-420.

Paulignan, Y., MacKenzie, C., Marteniuk, R., \& Jeannerod, M. (1991). Selective perturbation of visual input during prehension movements: 1. The effects of changing object position. Experimental Brain Research, 83, 502-512.

UNGERLEIDER, L. G., \& Mishrin, M. (1982). Two cortical visual systems. In D. J. Ingle, M. A. Goodale, \& R. J. W. Mansfield (Eds.), Analysis of visual behavior (pp. 549-586). Cambridge, MA: MIT Press.

(Manuscript received August 21, 1996; revision accepted for publication March 22, 1997.) 